\title{
Magnetic Properties of the Second Mott Lobe in Pairing Hamiltonians
}

\author{
M. J. Bhaseen, ${ }^{1}$ S. Ejima,${ }^{2}$ M. Hohenadler, ${ }^{3}$ A. O. Silver ${ }^{1}$ F. H. L. Essler,${ }^{4}$ H. Fehske, ${ }^{2}$ and B. D. Simons ${ }^{1}$ \\ ${ }^{1}$ University of Cambridge, Cavendish Laboratory, Cambridge, CB3 OHE, UK. \\ ${ }^{2}$ Institut für Physik, Ernst-Moritz-Arndt-Universität Greifswald, 17489 Greifswald, Germany. \\ ${ }^{3}$ Institut für Theoretische Physik und Astrophysik, Universität Würzburg, Germany. \\ ${ }^{4}$ The Rudolf Peierls Centre for Theoretical Physics, University of Oxford, Oxford, OX1 3NP, UK.
}

(Dated: October 18, 2018)

\begin{abstract}
We explore the Mott insulating state of single-band bosonic pairing Hamiltonians using analytical approaches and large scale density matrix renormalization group calculations. We focus on the second Mott lobe which exhibits a magnetic quantum phase transition in the Ising universality class. We use this feature to discuss the behavior of a range of physical observables within the framework of the 1D quantum Ising model and the strongly anisotropic Heisenberg model. This includes the properties of local expectation values and correlation functions both at and away from criticality. Depending on the microscopic interactions it is possible to achieve either antiferromagnetic or ferromagnetic exchange interactions and we highlight the possibility of observing the $\mathrm{E}_{8}$ mass spectrum for the critical Ising model in a longitudinal magnetic field.
\end{abstract}

PACS numbers: 37.10.Jk, 75.10.Jm, 75.10.Pq

\section{INTRODUCTION}

The observation of Bose-Einstein condensation (BEC) in dilute alkali gases [1, 2] has led to a wealth of activity linking ultra cold atoms research and condensed matter physics. The precise control over atomic interactions, and the use of optical lattices, offers valuable insights into the effects of strong correlations 3]. This is exemplified by experiments on the Bose-Hubbard model which reveal the quantum phase transition from a superfluid (SF) to a Mott insulator (MI) as the depth of the optical lattice is increased [4, 5]. Motivated by these advances, recent attention has been directed towards a variety of multicomponent systems, including spinor condensates [6 17 . and atomic mixtures 18 23. The possibility of novel behavior is greatly enhanced in the presence of these additional "spin" degrees of freedom, and routes to quantum magnetism have been proposed by exploiting internal hyperfine states 24] and using different atomic species 2528]. The decoupling of the electronic and nuclear spins in alkaline earth atoms has also been suggested as a way to realize quantum spin liquids and exotic magnetism based on the $\mathrm{SU}(\mathrm{N})$ groups 29 31].

In recent work some of the present authors suggested using atom-molecule mixtures as a route to the paradigmatic quantum Ising model 32, 33]. Motivated by studies of the BEC-BCS transition for bosons, both in the continuum limit 34 36] and on the lattice 37-40], we investigated the rich phase diagram of bosons interacting via Feshbach resonant pairing interactions in an optical lattice. Combining exact diagonalization (ED) on small systems with analytical predictions based on the strong coupling expansion, we provided evidence for an Ising quantum phase transition in the second Mott lobe. In contrast to previous numerical studies which advocated the presence of super-Mott behavior [39, 40], the Ising spectral gap indicates the absence of low lying superfluid excitations deep within the Mott phase [32, 33]. This conclusion gained further support in a recent comment [41] which shows the presence of exponential decay in the connected correlation functions. A detailed discussion of the superfluid properties [34 36] in one dimension (1D) was also provided in Ref. 42] using large scale density matrix renomalization group (DMRG) [43] and field theory techniques.

In view of the broad interest in these systems, and the technical issues surrounding Refs. [39, 40], we discuss the properties of the second Mott lobe in detail using analytical arguments and DMRG. To aid the comparison with these previous works we focus primarily on the homonuclear case with a single species of bosonic atom. We also begin by restricting the local Hilbert space for the atomic and molecular occupations [40]. In this reduced setting we determine both the locus of the antiferromagnetic Ising transition and the onset of superfluidity. We also investigate the atomic and molecular correlation functions within the MI and compare directly to the predictions of the quantum Ising model. As advocated in Refs. 32, 33] this provides a simple and intuitive framework in which to discuss the absence of super-Mott behavior 39 41].

Going beyond the restricted Hilbert space description we show that the same ideas apply. Interestingly, by tuning the microscopic interactions it is also possible to change the sign of the Ising exchange interaction from antiferromagnetic (AFM) to ferromagnetic (FM). In general these Ising Hamiltonians also contain an effective magnetic field [32, 33] and in 1D this will act as a confinement potential for the zero field excitations of the FM chain [4, 45]. This suggests the possibility of observing the non-trivial $\mathrm{E}_{8}$ mass spectrum of "meson" bound states for the critical FM Ising model in a longitudinal field [46 48]. As an extension of these results we also provide the magnetic Hamiltonian for Bose-Fermi mixtures.

The layout of this paper is as follows. In Secs. II and III we provide an introduction to the bosonic Feshbach Hamiltonian and discuss the mapping to the quantum 
Ising model. In Sec. IV we present a cross section of the phase diagram obtained by DMRG which displays both the Ising quantum phase transition and the onset of superfluidity. We confirm the Ising behavior within the Mott phase using results for the excitation gap and the entanglement entropy. In Sec. $\mathrm{V}$ we investigate the role of higher order terms in the strong coupling expansion. We discuss their impact on the non-universal properties of the phase diagram such as the curvature of the Ising phase boundary. In Sec.VI we examine the local expectation values within the Mott phase at and away from criticality. We move on to correlation functions in Secs. VII and VIII and discuss their relation to the Ising model. In Secs. [X] and $\mathrm{X}$ we turn our attention to softcore bosons and demonstrate the existence of both antiferromagnetic and ferromagnetic Ising transitions. We comment on the closely related fermionic problem in Appendix B. We conclude in Sec. XI and provide perspectives for further research.

\section{MODEL}

We consider the Hamiltonian 37 40

$$
\begin{array}{r}
H=\sum_{i \alpha} \epsilon_{\alpha} n_{i \alpha}-\sum_{\langle i j\rangle} \sum_{\alpha} t_{\alpha}\left(b_{i \alpha}^{\dagger} b_{j \alpha}+\text { H.c. }\right) \\
+\sum_{i \alpha \alpha^{\prime}} \frac{U_{\alpha \alpha^{\prime}}}{2}: n_{i \alpha} n_{i \alpha^{\prime}}:+H_{\mathrm{F}}
\end{array}
$$

describing bosons, $b_{i \alpha}$ hopping on a lattice with sites $i$ where $\alpha=a, m$ labels atoms and molecules and $n_{i \alpha}=$ $b_{i \alpha}^{\dagger} b_{i \alpha}$. Here, $\epsilon_{\alpha}$ are on-site potentials, $t_{\alpha}$ are hopping parameters, $\langle i j\rangle$ denotes summation over nearest neighbor bonds and $U_{\alpha \alpha^{\prime}}$ are interactions. We use normal ordering symbols to indicate $: n_{i \alpha} n_{i \alpha^{\prime}}:=n_{i \alpha}\left(n_{i \alpha}-1\right)$ for like species and $: n_{i \alpha} n_{i \alpha^{\prime}}:=n_{i \alpha} n_{i \alpha^{\prime}}$ for distinct species. For simplicity we assume that molecules are formed by $s$-wave Feshbach resonant interactions

$$
H_{\mathrm{F}}=g \sum_{i}\left(m_{i}^{\dagger} a_{i} a_{i}+\text { H.c. }\right)
$$

where $m_{i} \equiv b_{i m}$ and $a_{i} \equiv b_{i a}$; for recent work on the $p$-wave problem see Ref. [49]. An important feature of the Feshbach interaction (2) is that atoms and molecules are not separately conserved. However, the total, $N_{\mathrm{T}} \equiv \sum_{i}\left(n_{i a}+2 n_{i m}\right)$ is preserved. One may therefore work in the canonical ensemble with $\rho_{\mathrm{T}} \equiv N_{\mathrm{T}} / L$ held fixed, where $L$ is the number of lattice sites. In order to make contact with the previous literature 39 41] we consider only a single-band description in Eq. (11); for a discussion of higher band effects see Refs. [50 52]. As reported elsewhere the pairing Hamiltonian (11) has a rich phase diagram exhibiting both MI and SF phases 32 40, 42]. Most notably, the system displays a discrete $\mathbb{Z}_{2}$ symmetry breaking transition [34 36] between a paired molecular condensate (MC) and an atomic plus molecular condensate $(\mathrm{AC}+\mathrm{MC})$ phase [42]; for closely related

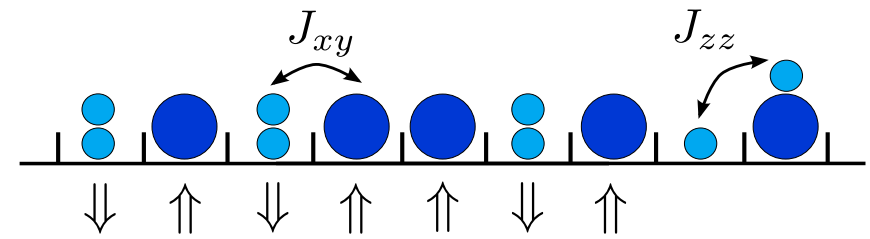

FIG. 1. (color online). Depiction of the second Mott lobe of the Hamiltonian (1) with $\rho_{\mathrm{T}}=2$ showing either a pair of atoms or a molecule on each site. These may be regarded as an effective spin-down $(\Downarrow)$ and spin-up (介) respectively. Second order virtual hopping processes lead to $J_{z z}$ interactions and an effective Ising model. XY exchange, $J_{x y}$, occurs at third order and involves interchanging a pair of atoms and a molecule. The Feshbach term (2) converts a pair of atoms ( $\Downarrow)$ into a molecule ( $\Uparrow)$ and therefore acts like a transverse field.

transitions in other models see also Refs. [53 57]. Here, our main focus will be on the MI phase. In particular, we shed further light on the magnetic characteristics of the second Mott lobe [32, 33]. We also discuss the connection to Ref. [1] and earlier numerical studies [39, 40]. In order to facilitate our numerical simulations we consider the restricted Hamiltonian

$$
H_{\mathcal{P}}=\mathcal{P} H \mathcal{P},
$$

where the projection operator $\mathcal{P}$ projects on to the restricted local Hilbert space with a maximum of $r_{a}$ atoms and $r_{m}$ molecules per site. We begin our discussion in Sec. III by setting $r_{a}=2$ and $r_{m}=1$ as used in Ref. [40]. As we will see, the essential characteristic features of the phase diagram are readily gleaned from this limiting case. We move on to the more general problem with canonical softcore bosons in Secs. [X] and X] Throughout this manuscript we use the value of $U_{a m}$ to set the overall energy scale; in Figs. 2, 3, 4 and Figs. 6, 7, 8 we set $U_{a m}=1$, and in the remaining figures we set $U_{a m}=4$ in order to descend deeper in to the Mott phase.

\section{SECOND MOTT LOBE}

A convenient way to describe the second Mott lobe with $\rho_{\mathrm{T}}=2$ is via an effective spin model derived within the strong coupling expansion; see Fig. 1. Introducing effective spins $|\Downarrow\rangle \equiv|2 ; 0\rangle / \sqrt{2}$ and $|\Uparrow\rangle \equiv|0 ; 1\rangle$ in the occupation basis $\left|n_{a} ; n_{m}\right\rangle$ one obtains the effective spin$1 / 2$ quantum Ising model 32, 33.

$$
H \simeq J_{z z} \sum_{i} S_{i}^{z} S_{i+1}^{z}+h \sum_{i} S_{i}^{z}+\Gamma \sum_{i} S_{i}^{x}+C+\mathcal{O}\left(t^{3}\right),
$$

where we work to second order in the hopping parameters. The spin operators are given by

$$
S^{+}=\frac{m^{\dagger} a a}{\sqrt{2}}, \quad S^{-}=\frac{a^{\dagger} a^{\dagger} m}{\sqrt{2}}, \quad S^{z}=\frac{\left(n_{m}-n_{a} / 2\right)}{2},
$$

and $S^{ \pm} \equiv S^{x} \pm i S^{y}$. The factors of $\sqrt{2}$ arise from the action of the Bose operators on the basis states. 
Equivalently, since $n_{a}+2 n_{m}=2$, one may also write $S^{z}=\left(1-n_{a}\right) / 2$ or $S^{z}=\left(2 n_{m}-1\right) / 2$. As indicated in Fig. 1, the first term in Eq. (4) arises from the virtual hopping processes of atoms and molecules on to neighboring sites and corresponds to an effective magnetic exchange interaction, $J_{z z}$. The second term in Eq. (44) reflects the energetic detuning between atoms and molecules and corresponds to an effective longitudinal magnetic field, $h$. The third term in Eq. (4) corresponds directly to the Feshbach term in Eq. (2). Indeed, it is readily seen from the definition (5) that $S^{+}$converts two atoms $(\Downarrow)$ into a molecule $(\Uparrow)$ and therefore acts like a spin raising operator. It follows that the Feshbach term (2) acts like a transverse field in the $x$-direction with

$$
\Gamma \equiv 2 g \sqrt{2} .
$$

The overall structure of the magnetic Hamiltonian (4) is generic to the second Mott lobe with $\rho_{\mathrm{T}}=2$. However, the coefficients depend on the specific Hilbert space restriction. For the Hamiltonian (3) with restriction parameters $r_{a}=2$ and $r_{m}=1$ one obtains [32, 33]

$$
J_{z z}=\frac{4 t_{a}^{2}}{U_{a m}-U_{a a}}+\frac{t_{m}^{2}}{U_{a m}},
$$

and

$$
h=\epsilon_{m}-2 \epsilon_{a}-U_{a a} .
$$

The constant offset is given by

$$
C=L\left(\frac{\epsilon_{m}}{2}+\epsilon_{a}+\frac{U_{a a}}{2}-\frac{z J_{z z}}{8}\right),
$$

where $z$ is the cubic lattice coordination and $z=2$ in 1D. In general there will also be additional contributions to these coefficients arising from higher order terms in the strong coupling expansion. As we will discuss in more detail below similar results also hold for canonical softcore atoms and molecules but with modified coefficients. This is due to the presence of additional intermediate states that are explored in the virtual hopping processes. Nonetheless, this truncation of the Hilbert space is useful for initial numerical simulations, and is also used in Ref. [40]. In addition the principal features of the magnetic description are more readily exposed. We will return to canonical softcore bosons in Secs. [X] and X

\section{PHASE DIAGRAM AND THE ANTIFERROMAGNETIC ISING TRANSITION}

In Refs. [32, 33] we provided a variety of evidence for an Ising quantum phase transition in the closely related heteronuclear generalization of Hamiltonian (11). However, due to the small system sizes accessible by exact diagonalization, a complete elucidation of the phase diagram was not possible. In addition correlation functions were out of reach. In particular, it was not possible to track the Ising quantum phase transition throughout the second Mott lobe, or to accurately delimit the onset of superfluidity. We address this situation for the homonuclear case by using DMRG on large systems. We keep up to 3000 density matrix states so that the discarded weight is less than $10^{-10}$. Following Ref. [40] we implement the model (3) as a two-leg ladder system, where the atoms and molecules reside on opposite legs of the ladder. In this representation the Feshbach term (2) corresponds to hopping along the rungs, and extreme care must be taken for small values of $g$. In Fig. 2 we present a cross sec-

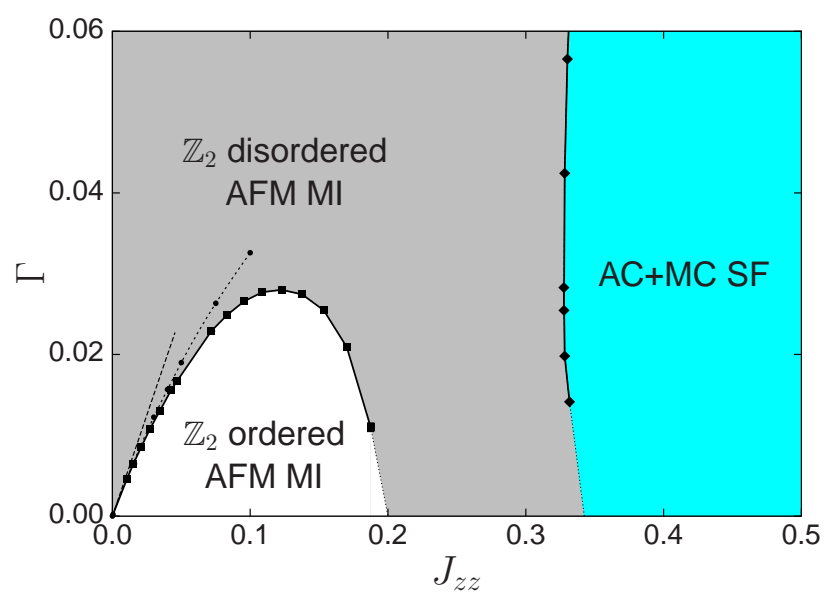

FIG. 2. (color online). Phase diagram of the 1D Hamiltonian (3) with $\rho_{\mathrm{T}}=2$ obtained by DMRG with up to $L=256$ sites. We restrict the local Hilbert space to a maximum of one molecule and two atoms per site with $r_{m}=1$ and $r_{a}=2$, and set $\epsilon_{a}=\epsilon_{m}=0, U_{a a}=0, U_{a m}=1$, corresponding to $h=0$. We set $t_{a}=2 t_{m}=t$ and vary $t$ to obtain different values of $J_{z z}=17 t^{2} / 4$ corresponding to antiferromagnetic (AFM) exchange. This cross section shows a $\mathbb{Z}_{2}$ ordered AFM MI, a $\mathbb{Z}_{2}$ disordered AFM MI, and an AC+MC SF. The filled squares are obtained from the vanishing of the first excitation gap, $\Delta_{1} \equiv E_{1}-E_{0}$ corresponding to an Ising quantum phase transition within the MI; the solid line is a spline fit which is extrapolated down to $\Gamma=0$. The dashed line corresponds to the strong coupling result, $\Gamma_{\mathrm{c}}=J_{z z} / 2$ which follows from the Hamiltonian (4) with $h=0$. The dotted line is obtained by ED of Hamiltonian (4) including the subleading XY exchange terms, $J_{x y}=-0.46 J_{z z}^{3 / 2}$ which arise at higher order in the strong coupling expansion. The diamonds are obtained from the vanishing of the two-particle gap, $E_{2 g} \equiv \mu_{+}-\mu_{-}$where $\mu_{ \pm}= \pm\left[E_{0}\left(L, N_{\mathrm{T}} \pm 2\right)-E_{0}\left(L, N_{\mathrm{T}}\right)\right]$ and up to $L=128$, indicating the onset of an $\mathrm{AC}+\mathrm{MC}$ superfluid.

tion of the phase diagram for the Hamiltonian (3) with $r_{a}=2$ and $r_{m}=1$. In this approach the Hilbert space restriction parameters $r_{\alpha}$ and the interactions $U_{\alpha \alpha^{\prime}}$ are treated independently. For ease of exposition we begin by setting $\epsilon_{a}=\epsilon_{m}=U_{a a}=0$ and $U_{a m}=1$, where our choice of parameters is motivated by the simplest case with zero magnetic field as given by Eq. (8). In addition, for small hopping parameters $t_{\alpha}$, and large $U_{a m}$, one may set $U_{a a}=0$ in Eq. (7) without loss of generality or conflict with the strong coupling expansion. We 
will incorporate the effects of finite $U_{a a}$ in our subsequent discussion.

The phase diagram in Fig. 2 contains three distinct phases. $\mathrm{A} \mathbb{Z}_{2}$ disordered $\mathrm{MI}$ with vanishing staggered magnetization $\sum_{i}(-1)^{i}\left\langle S_{i}^{z}\right\rangle / L=0$, a $\mathbb{Z}_{2}$ ordered MI with a finite staggered magnetization and long range antiferromagnetic correlations, and an $\mathrm{AC}+\mathrm{MC}$ superfluid with both atomic and molecular power law superfluidity. The additional MC phase with only molecular superfluidity is absent in this cross section of the phase diagram due to our choice of parameters; for more details of the superfluid phases see Ref. [42]. In this manuscript our main focus is on the MI phase. In particular, we see that the magnetic phase boundary bends over quite considerably due to higher order terms in the strong coupling expansion. Nonetheless, the quantum phase transition remains in the Ising universality class. For example, the Ising character of this transition is supported by Fig. 3 which shows the characteristic linear variation of the excitation gap [58]

$$
\Delta=\left|\Gamma-\Gamma_{\mathrm{c}}\right|,
$$

on passing through the transition in the scaling regime. The Ising character is also confirmed by DMRG results

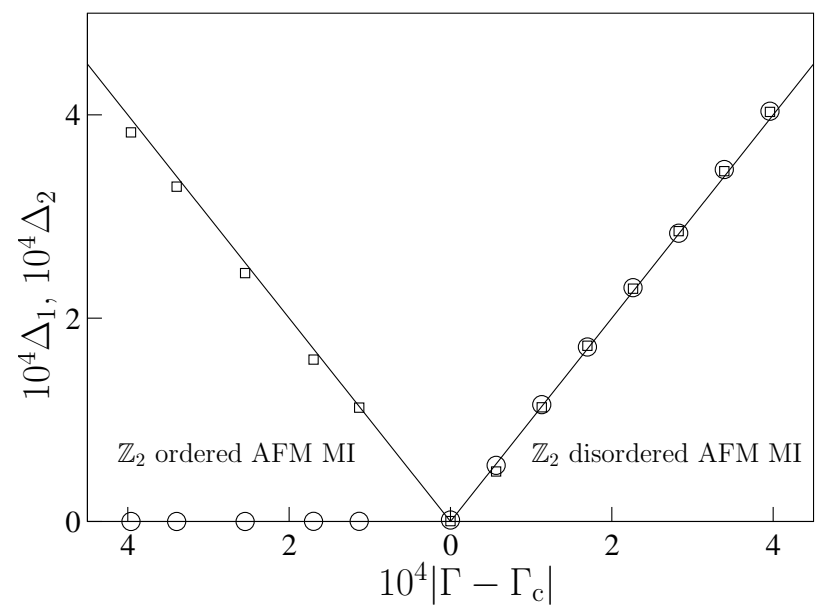

FIG. 3. DMRG results for the excitation gaps, $\Delta_{1} \equiv E_{1}-$ $E_{0}$ (circles) and $\Delta_{2} \equiv E_{2}-E_{0}$ (squares), for the bosonic Hamiltonian (3) with open boundaries and up to $L=256$ sites. We pass through the Ising quantum phase transition shown in Fig. 2 with $t=0.05$ and $J_{z z} \approx 0.01$. The transition occurs at $\Gamma_{\mathrm{c}} \approx 4.6 \times 10^{-3}$ which is slightly below the strong coupling result, $\Gamma_{\mathrm{c}}=J_{z z} / 2 \approx 5.3 \times 10^{-3}$. This reflects the curvature of the Ising transition shown in Fig. 2 The solid lines indicate the linear gap, $\Delta=\left|\Gamma-\Gamma_{\mathrm{c}}\right|$, corresponding to the Ising critical exponent, $\nu=1$.

for the entanglement entropy as shown in Fig. 4. For a block of length $l$ in a system of length $L$, the von Neumann entropy is given by $S_{L}(l)=-\operatorname{Tr}_{l}\left(\rho_{l} \ln \rho_{l}\right)$, where $\rho_{l}=\operatorname{Tr}_{L-l}(\rho)$ is the reduced density matrix. In a critical system with periodic boundaries one obtains [59 62 [ 62 ]

$$
S_{L}(l)=\frac{c}{3} \ln \left[\frac{2 L}{\pi} \sin \left(\frac{\pi l}{L}\right)\right]+s_{1},
$$
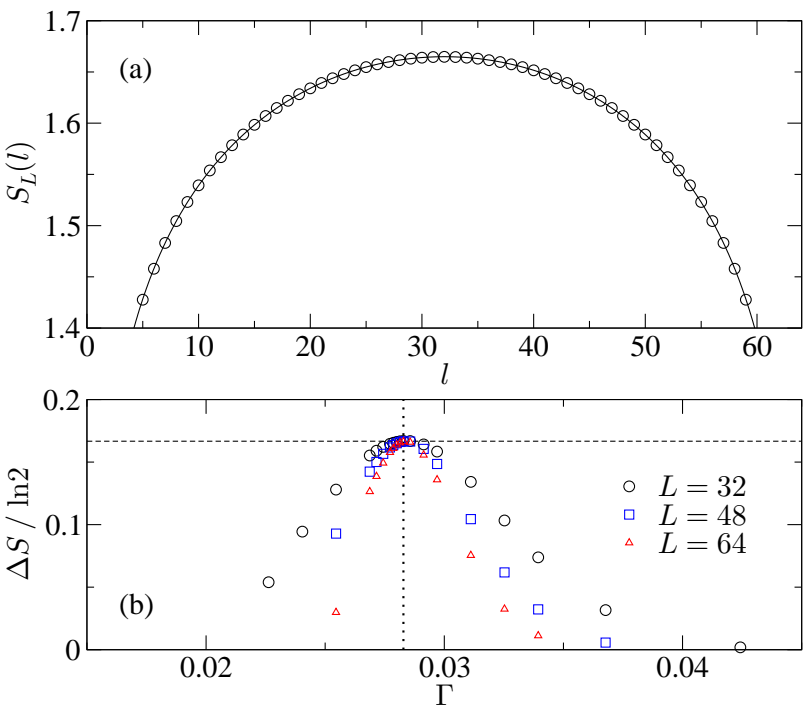

FIG. 4. (a) DMRG results for the entanglement entropy, $S_{L}(l)$, at the Ising transition within the MI for a subsystem of length $l$ in an periodic chain with $L=64$. We use the same parameters as in Fig. 2 and set $t=0.17$ corresponding to $J_{z z} \approx 0.12$ and $\Gamma_{c} \approx 0.028$. The solid line is a fit to Eq. (11). The extracted value of the central charge $c=0.50(2)$ is consistent with a magnetic transition in the Ising universality class. (b) The entanglement entropy difference $\Delta S(L)$ for $t=0.17$ shows a peak at the Ising transition. The peak height corresponds to $c=1 / 2$ as indicated by the dashed lines.

where $s_{1}$ is a non-universal constant and $c$ is the central charge. As shown in Fig. 4(a) the results are in excellent agreement with an Ising quantum phase transition with $c=1 / 2$. In particular, this continues to hold further out in the Mott phase where the strong coupling analysis no longer strictly applies. Further evidence for this may be seen from the entanglement entropy difference [42, 63]

$$
\Delta S(L) \equiv S_{L}(L / 2)-S_{L / 2}(L / 4)
$$

which exhibits a peak on transiting through the magnetic transition as shown in Fig. 4(b). At criticality $\Delta S=\frac{c}{3} \ln 2+\ldots$ 59 62 and the peak height is in good agreement with $c=1 / 2$. It is interesting to note that a naive spline fit to the currently available DMRG data for the Ising phase boundary shown in Fig. 2 terminates within the MI. This tentatively suggests that a magnetic transition may exist even in the absence of the Feshbach term (2). Unfortunately, it is difficult to gain a quantitative handle on the character of this feature, which occurs for intermediate hopping strengths and vanishing Feshbach coupling, using our current implementation of DMRG. Moreover, this $\Gamma=0$ limit differs somewhat from other investigations of the two-component BoseHubbard model [64, 65], since we keep the total density, $\rho_{\mathrm{T}}=\sum_{i}\left(n_{i a}+2 n_{i m}\right) / L=2$ held fixed, with two atoms and one molecule per site. It would be instructive to explore this limit in future work. 


\section{HIGHER ORDER CONTRIBUTIONS IN THE STRONG COUPLING EXPANSION}

In the discussion above we have presented a variety of large scale DMRG results in favor of an Ising quantum phase transition taking place within the second Mott lobe [32, 33]. In particular we have argued that the Ising character of the quantum phase transition persists throughout an extended region of the phase diagram as shown in Fig. 2. Nonetheless, it is important to bear in mind that the explicit Ising Hamiltonian given in Eq. (4) is derived by means of the strong coupling, $t / U$ expansion. It is therefore tailored towards a quantitative description of the bosonic Hamiltonian (11) deep within the Mott lobe, as supported by our DMRG results. At larger values of the hopping parameters departures from the strong coupling result (41) are to be expected. This is evident from the deviation of the phase boundary from the asymptotic result, $\Gamma_{\mathrm{c}}=J_{z z} / 2$ as shown in Fig. 2. Carrying out the strong coupling expansion to third order in the hopping parameters one must supplement the Hamiltonian (44) with the additional XY exchange terms:

$$
\Delta H=\frac{J_{x y}}{2} \sum_{i}\left(S_{i}^{+} S_{i+1}^{-}+S_{i}^{-} S_{i+1}^{+}\right),
$$

where $S^{ \pm} \equiv S^{x} \pm i S^{y}$, and

$$
J_{x y}=-\frac{4 t_{a}^{2} t_{m}}{U_{a m}-U_{a a}}\left(\frac{1}{U_{a m}}+\frac{1}{U_{a m}-U_{a a}}\right) .
$$

One thus obtains a strongly anisotropic XXZ Heisenberg Hamiltonian in both a longitudinal and transverse field. For the parameters chosen in Fig. 2, $J_{z z}=17 t^{2} / 4$ and $J_{x y}=-4 t^{3}$. This yields the XXZ anisotropy parameter, $\delta \equiv J_{x y} / J_{z z}=-16 t / 17$ corresponding to $\delta \approx-0.46 \sqrt{J_{z z}}$. Within the MI shown in Fig. 2 this gives $-0.27 \lesssim \delta<0$. The system remains in the strongly anisotropic regime and Ising criticality is expected to persist throughout this cross section of the MI. Nonetheless, the XY contributions (13) modify the location of the Ising quantum phase transition as shown in Fig. 2, As we will discuss in Sec. VII such terms also influence the nonuniversal amplitudes in the correlation functions whilst preserving the universal critical exponents.

\section{LOCAL EXPECTATION VALUES}

Having provided numerical evidence for an Ising quantum phase transition in the second Mott lobe of the Hamiltonian (11) we turn our attention to the local expectation values. The order parameter of the antiferromagnetic transverse field Ising model (4) is the staggered magnetization $(-1)^{i}\left\langle S_{i}^{z}\right\rangle$. In the thermodynamic limit with $h=0$ and $\Gamma<\Gamma_{\mathrm{c}}$ this is given by [66, 67]

$$
(-1)^{i}\left\langle S_{i}^{z}\right\rangle=\frac{1}{2}\left[1-\left(\frac{\Gamma}{\Gamma_{\mathrm{c}}}\right)^{2}\right]^{\beta},
$$

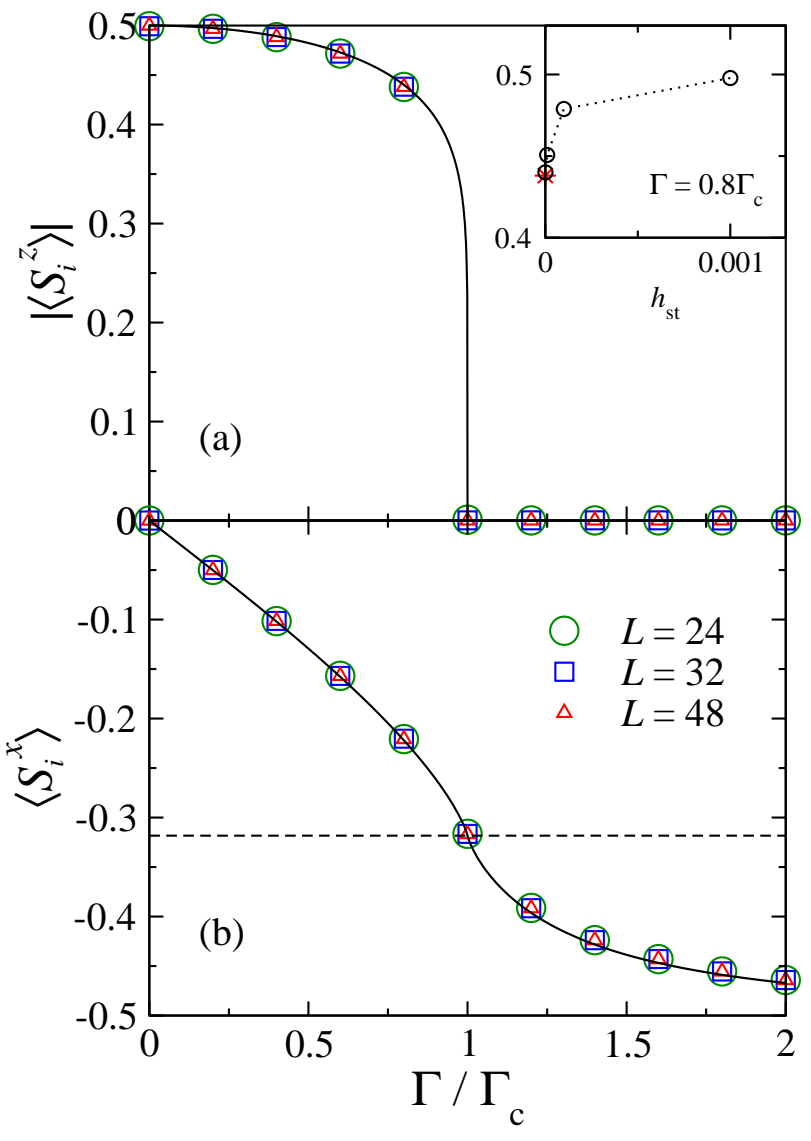

FIG. 5. (color online). Local expectation values deep within the Mott phase obtained by DMRG for the bosonic Hamiltonian (3) with periodic boundaries and restriction parameters $r_{m}=1$ and $r_{a}=2$. In order to demonstrate the broader validity of our results, whilst suppressing higher order terms in the strong coupling expansion, we choose different parameters to those used in Fig. 22 We set $\epsilon_{a}=0$ and $\epsilon_{m}=U_{a a}=3.8$, corresponding to $h=0$ and take $U_{a m}=4$ and $t=0.005$. (a) Staggered magnetization $\left|\left\langle S_{i}^{z}\right\rangle\right|$. The theoretical result for the transverse field Ising model is given by Eq. (15) and is indicated by the solid line. Inset: The application of a small staggered field $h_{s t}$ yields the same results as in panel (a) in the limit $h_{\mathrm{st}} \rightarrow 0$. (b) Transverse magnetization $\left\langle S_{i}^{x}\right\rangle$. The solid line shows the Ising behavior given by Eq. (16). The dashed line corresponds to $\left\langle S_{i}^{x}\right\rangle=-1 / \pi$ which holds at criticality.

where $\beta=1 / 8$ is the Ising critical exponent; in the disordered phase with $\Gamma>\Gamma_{\mathrm{c}}$ the order parameter vanishes. In order to verify this characteristic behavior in the bosonic Hamiltonian (3) we choose parameters deep within the Mott phase. As shown in Fig. [5(a) our DMRG results are in excellent agreement with Eq. (15). This confirms that any higher order corrections to the Ising description (4) are small. In a similar fashion the transverse magnetization is given by [66, 67]

$$
\left\langle S_{i}^{x}\right\rangle=-\int_{0}^{\pi} \frac{d k}{2 \pi} \frac{2 \Gamma+J_{z z} \cos k}{\sqrt{4 \Gamma^{2}+J_{z z}^{2}+4 \Gamma J_{z z} \cos k}} .
$$


This dependence is confirmed in Fig. 5 (b) both at and away from criticality. Note that at the critical point where $\Gamma=\Gamma_{\mathrm{c}}$ the expectation value $\left\langle S_{i}^{x}\right\rangle=-1 / \pi$ is non-vanishing due to the transverse field. We have also checked the consistency of our DMRG results by applying a small staggered field $h_{\text {st }}$ to the bosonic Hamiltonian (3), $\Delta H_{\mathrm{st}}=-h_{\mathrm{st}} \sum_{i}(-1)^{i} S_{i}^{z}$, where $S_{i}^{z}$ is given by Eq. (5). In the limit $h_{\mathrm{st}} \rightarrow 0$ this replicates the effect of spontaneous symmetry breaking in our finite size simulations. The results converge to the same values as for $h_{\mathrm{st}}=0$;

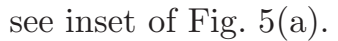

Having established the validity of the explicit Ising Hamiltonian (44) deep within the Mott phase, it is instructive to see how this leading order behavior is modified as one moves out towards the MI-SF boundary. In Fig. 6(a) we show the evolution of the local magnetization $\left\langle S_{i}^{z}\right\rangle$ with increasing hopping parameters within the $\mathbb{Z}_{2}$ disordered phase shown in Fig. 2. Deep within the MI, $\left\langle S_{i}^{z}\right\rangle=0$ as one would expect for the disordered phase of the transverse field Ising model (4) with $h=0$. However, at larger values of the hopping parameters a finite uniform magnetization develops as indicated by the plateaus in Fig.6(a). This is due to the presence of higher order terms in the strong coupling expansion. In general such contributions are expected to induce corrections to the leading order coefficients, $J_{z z}$ and $C$, and the magnetic field, $h$, given in Eqs. (7) - (9). However, it is evident from Fig. 6(b) that this uniform contribution to the magnetization remains significantly below the saturation value of $\left\langle S_{i}^{z}\right\rangle=-1 / 2$, indicating that the leading order description (4) is a useful starting point in the broader phase diagram. Indeed, the staggered magnetization remains zero in the bulk of the system, as may be seen from the absence of oscillations in Fig. 6(a). As such we remain in the disordered phase of an Ising antiferromagnet, albeit in the presence of an increasing uniform effective magnetic field. More generally, one may also transit through the $\mathbb{Z}_{2}$ ordered region shown in Fig. 2 As indicated in Figs. 6) (c) and (d) this results in the onset of antiferromagnetic oscillations in the local magnetization. In Fig. 6(e) we plot the evolution of the corresponding uniform and staggered magnetizations. The region of support of the staggered component is in agreement with the $\mathbb{Z}_{2}$ ordered phase inferred from the gap data in Fig. 2 .

\section{CORRELATION FUNCTIONS}

Having discussed the local expectation values we now consider correlation functions. In order to orient the discussion we first recall the expected theoretical behavior at the critical point of the antiferromagnetic Ising model in a transverse field, where $\Gamma_{\mathrm{c}}=J_{z z} / 2$ and $h=0$. In the absence of a longitudinal field the asymptotic longitudinal correlation function decays as a power law 66.

$$
\left\langle S_{i}^{z} S_{i+n}^{z}\right\rangle \sim(-1)^{n} \mathcal{B} n^{-\eta}+\ldots,
$$
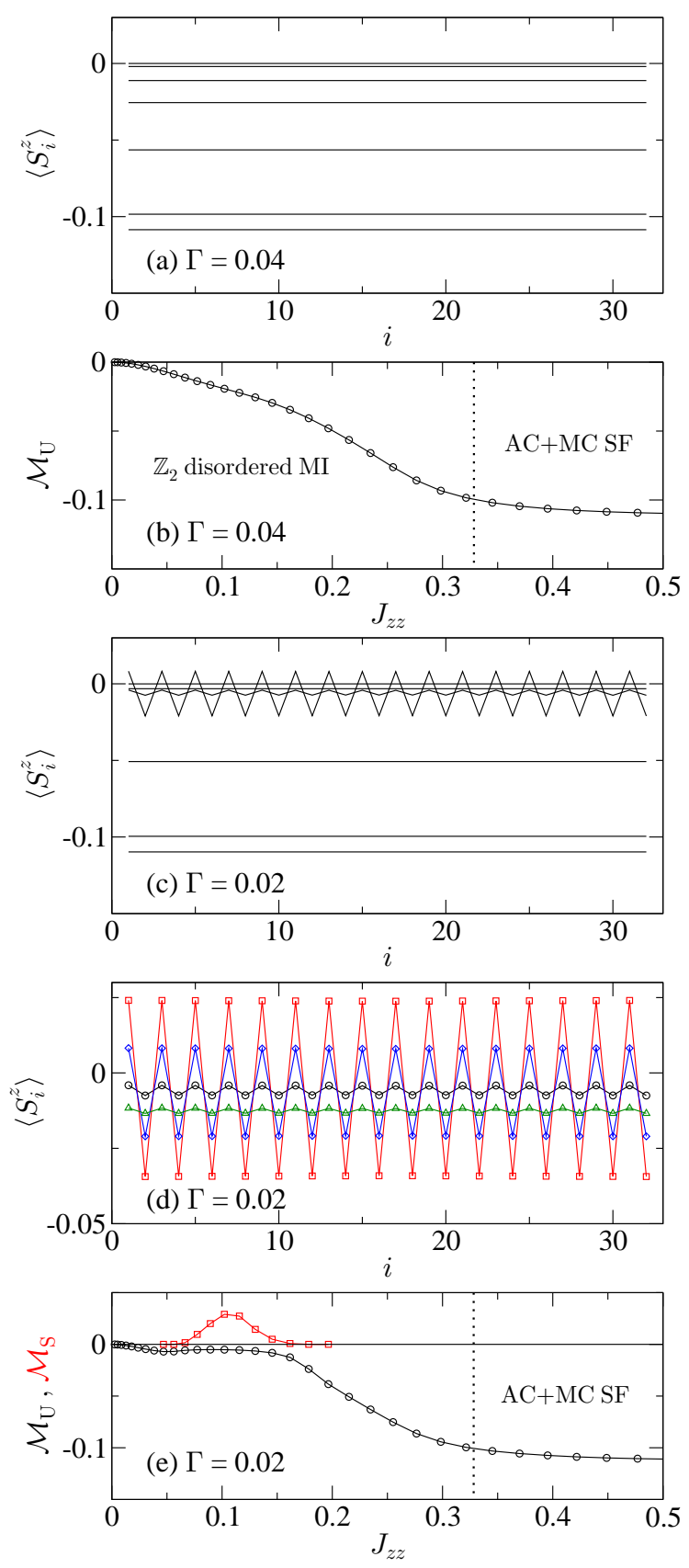

FIG. 6. (color online). (a) DMRG results for the local magnetization $\left\langle S_{i}^{z}\right\rangle$ for the parameters used in Fig. 2 with $h=0$ and $\Gamma=0.04$. We set $t=0.025,0.075,0.125, \ldots, 0.325$ from top to bottom with $L=32$ and periodic boundaries. The plateaus correspond to the development of a uniform magnetization whilst the staggered magnetization remains zero. (b) Uniform magnetization $\mathcal{M}_{\mathrm{U}}=\sum_{i}\left\langle S_{i}^{z}\right\rangle / L$. The non-zero value is attributed to higher order terms in the strong coupling expansion. These may modify the leading order magnetic field given in Eq. (8). The dotted line shows the approximate location of the $\mathrm{MI}$ to $\mathrm{AC}+\mathrm{MC}$ transition obtained from the gap data in Fig. 2 (c) We set $\Gamma=0.02$ and use the same values of the remaining parameters as in panel (a). The oscillations correspond to the onset of the $\mathbb{Z}_{2}$ ordered phase in Fig. 2. (d) Antiferromagnetic oscillations in the $\mathbb{Z}_{2}$ ordered phase with $t=0.125$ (circles), $t=0.155$ (squares), $t=0.175$ (diamonds) and $t=0.195$ (triangles). (e) Uniform magnetization $\mathcal{M}_{\mathrm{U}}$ (circles), and staggered magnetization $\mathcal{M}_{\mathrm{S}}=\sum_{i}(-1)^{i}\left\langle S_{i}^{z}\right\rangle / L$ (squares) for $\Gamma=0.02$. 
where $\eta=1 / 4$ is the Ising pair correlation exponent, $\mathcal{B}=\mathcal{A}^{-3} 4^{-1} 2^{1 / 12} e^{1 / 4} \simeq 0.161$ is a constant prefactor, and $\mathcal{A} \simeq 1.2824$ is the Glaisher-Kinkelin constant. In a similar fashion, the transverse correlators also exhibit power law behavior at criticality [66]

$$
\left\langle S_{i}^{x} S_{i+n}^{x}\right\rangle \sim\left\langle S_{i}^{x}\right\rangle^{2}+(2 \pi n)^{-2}+\ldots,
$$

where $\left\langle S_{i}^{x}\right\rangle=-1 / \pi$ at the transition and

$$
\left\langle S_{i}^{y} S_{i+n}^{y}\right\rangle \sim-(-1)^{n}(\mathcal{B} / 4) n^{-9 / 4}+\ldots
$$

As suggested by equation (18), due to the finite value of $\left\langle S_{i}^{x}\right\rangle=-1 / \pi$ at criticality one must consider the connected correlation function $\left\langle S_{i}^{x} S_{i+n}^{x}\right\rangle-\left\langle S_{i}^{x}\right\rangle\left\langle S_{i+n}^{x}\right\rangle$ in order to see power law behavior. To establish this dependence in the bosonic Hamiltonian (3) we perform DMRG calculations with open boundaries and up to $L=512$ sites. As shown in Fig. (7) at $\Gamma=\Gamma_{\mathrm{c}}$ the data are consistent with power law behavior in the bulk of the system away from the sample boundaries. In addition the data show long range order in $\left|\left\langle S_{i}^{z} S_{i+n}^{z}\right\rangle\right|$ for $\Gamma<\Gamma_{\mathrm{c}}$, and a finite correlation length for $\Gamma>\Gamma_{\mathrm{c}}$. Although this provides direct evidence for a quantum phase transition, a quantitative determination of the Ising critical exponents requires a more detailed finite size scaling analysis of the data. This is most readily achieved using periodic boundary conditions. We recall that in a finite size critical system with periodic boundary conditions, the two-point function of a primary field $\mathcal{O}$ may be obtained by conformal transformation of the strip geometry [68]:

$$
\left\langle\mathcal{O}\left(r_{1}\right) \mathcal{O}\left(r_{2}\right)\right\rangle_{L}=\mathcal{N}\left[\frac{\pi}{L \sin \left(\frac{\pi r}{L}\right)}\right]^{a} .
$$

Here $a$ is the critical exponent in the thermodynamic limit and $\mathcal{N}$ is the prefactor: $\left\langle\mathcal{O}\left(r_{1}\right) \mathcal{O}\left(r_{2}\right)\right\rangle_{\infty}=\mathcal{N} r^{-a}$. It follows from equation (20) that the rescaled combination

$$
L^{a}\left\langle\mathcal{O}\left(r_{1}\right) \mathcal{O}\left(r_{2}\right)\right\rangle_{L}=f_{a}(r / L)
$$

is a prescribed scaling function

$$
f_{a}(x)=\mathcal{N}\left[\frac{\pi}{\sin (\pi x)}\right]^{a},
$$

of the reduced separation $x=r / L$. As shown in Figs. 8 and 9, the rescaled critical two-point functions

$$
\begin{aligned}
& \mathcal{F}_{z}(n / L) \equiv L^{1 / 4}\left|\left\langle S_{i}^{z} S_{i+n}^{z}\right\rangle_{L}\right| \\
& \mathcal{F}_{y}(n / L) \equiv L^{9 / 4}\left|\left\langle S_{i}^{y} S_{i+n}^{y}\right\rangle_{L}\right| \\
& \mathcal{F}_{x}(n / L) \equiv L^{2}\left[\left\langle S_{i}^{x} S_{i+n}^{x}\right\rangle-\left\langle S_{i}^{x}\right\rangle\left\langle S_{i+n}^{x}\right\rangle\right]_{L}
\end{aligned}
$$

all show striking data collapse over the entire system length, showing clear indications of Ising criticality. Deep in the Mott phase the resulting scaling functions are in excellent agreement with the theoretical results for the lattice Ising model (4) in finite size geometry, as indicated in Fig. 9. This includes both the universal critical

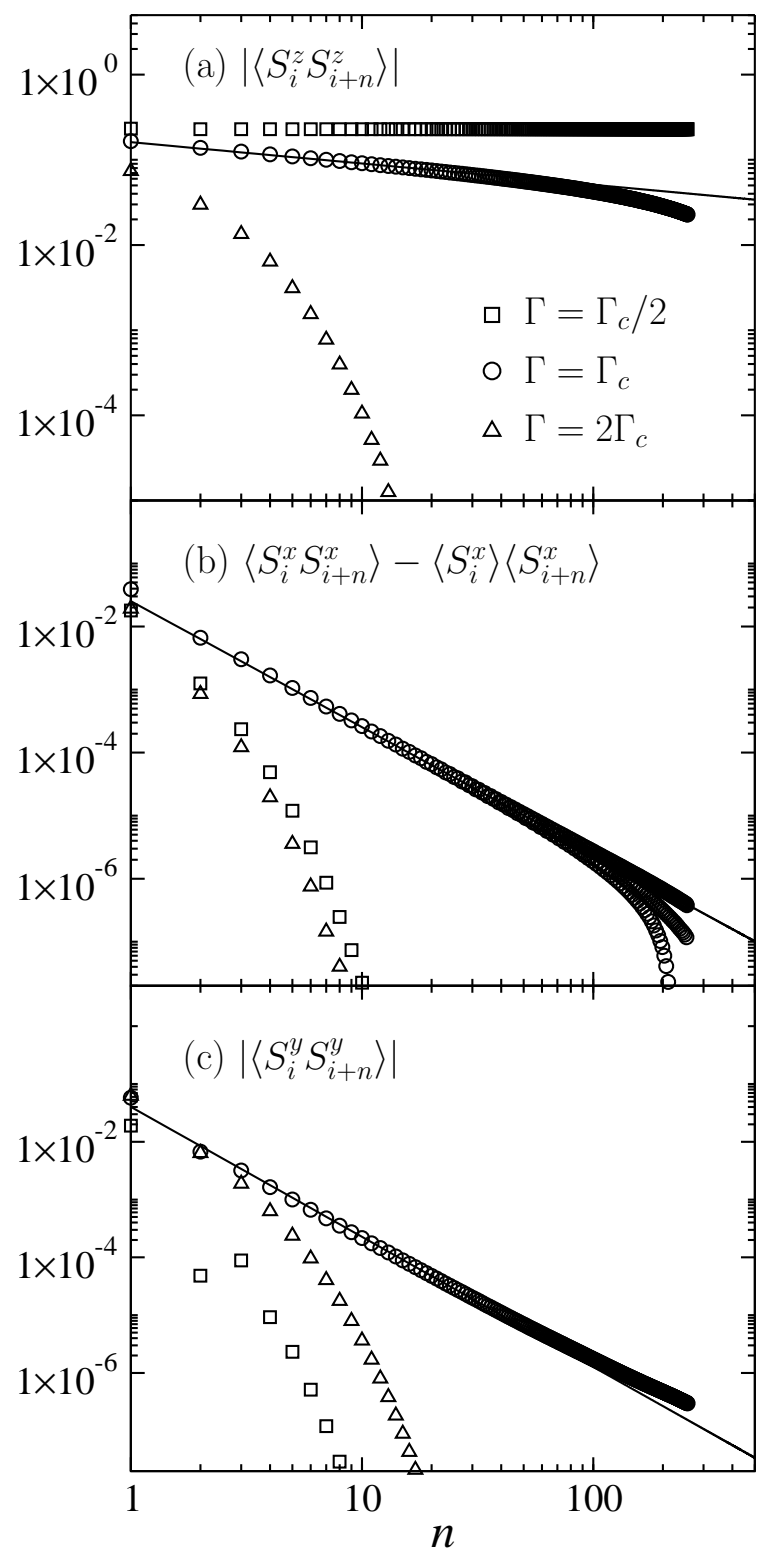

FIG. 7. Correlation functions within the second Mott lobe of the bosonic Hamiltonian (3) obtained by DMRG with $L=512$ and open boundaries. We use the same parameters as in Fig. 3 The data are consistent with an Ising quantum phase transition between a magnetic state with long range order and a disordered state with a finite correlation length. The solid lines correspond to the critical two-point functions in Eqs. (17), (18) and (19). A quantitative demonstration of the Ising critical exponents is shown in Fig. 8 using periodic boundaries and finite size scaling.

exponents, $a$ and the non-universal amplitude prefactors, $\mathcal{N}$ taken from Eqs. (17), (18) and (19). Further out in the Mott phase, the non-universal prefactors are influenced by higher order terms in the strong coupling expansion as discussed in Sec. $\mathrm{V}$, but the universal Ising exponents are robust to these perturbations; see Fig. 8

Having confirmed the presence of a line of critical Ising 


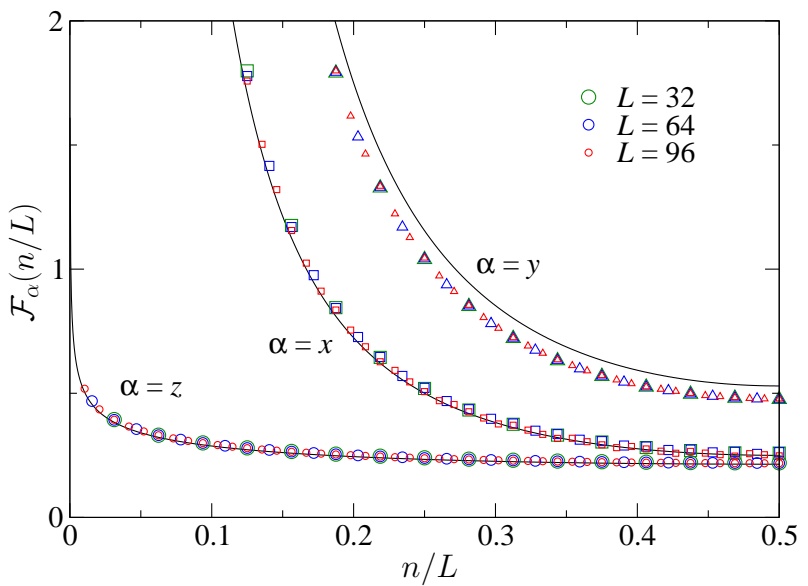

FIG. 8. (color online). DMRG results for the rescaled bosonic correlation functions $L^{1 / 4}\left|\left\langle S_{i}^{z} S_{i+n}^{z}\right\rangle_{L}\right|$ (circles), $L^{2}\left[\left\langle S_{i}^{x} S_{i+n}^{x}\right\rangle-\right.$ $\left.\left\langle S_{i}^{x}\right\rangle\left\langle S_{i+n}^{x}\right\rangle\right]_{L}$ (squares) and $L^{9 / 4}\left|\left\langle S_{i}^{y} S_{i+n}^{y}\right\rangle_{L}\right|$ (triangles) with periodic boundaries at criticality for the parameter set of Fig. 7 with $t=0.05$. The data collapse over the entire system length with the Ising critical exponents. The non-universal prefactors differ slightly from the theoretical predictions of the lattice Ising model (4) as indicated by the solid lines. This is due to the presence of small additional XY contributions to the Ising description. By descending deeper into the Mott lobe one obtains a complete quantitative agreement including the non-universal prefactors as shown in Fig. 9.

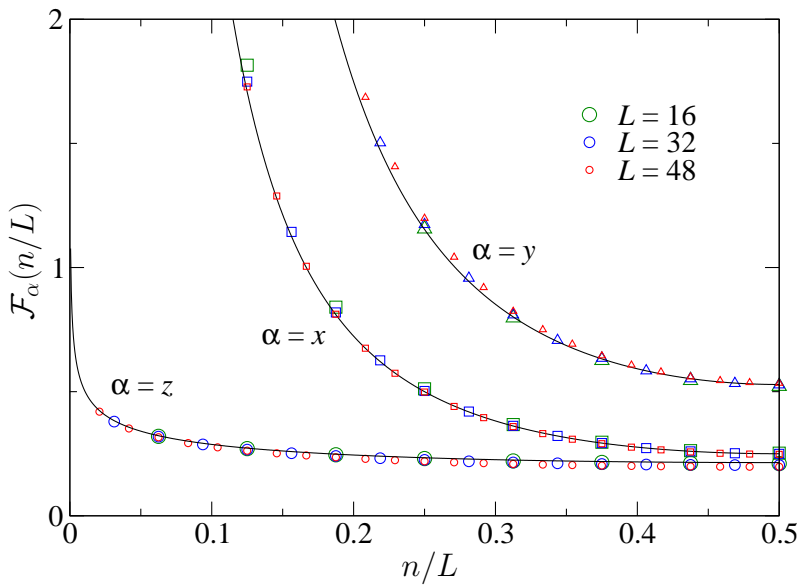

FIG. 9. (color online). DMRG results for the rescaled bosonic correlation functions $L^{1 / 4}\left|\left\langle S_{i}^{z} S_{i+n}^{z}\right\rangle_{L}\right|$ (circles), $L^{2}\left[\left\langle S_{i}^{x} S_{i+n}^{x}\right\rangle-\right.$ $\left.\left\langle S_{i}^{x}\right\rangle\left\langle S_{i+n}^{x}\right\rangle\right]_{L}$ (squares) and $L^{9 / 4}\left|\left\langle S_{i}^{y} S_{i+n}^{y}\right\rangle_{L}\right|$ (triangles) with periodic boundaries at criticality for the parameter set of Fig. 5 with $t=0.005$. The data show clear scaling collapse over the entire system length. The theoretical results for the lattice Ising model (4) in finite size geometry are indicated by solid lines. The data are in excellent agreement with both the universal Ising critical exponents and the non-universal amplitudes in Eqs. (17), (18) and (19).

correlations within the second Mott lobe of the bosonic Hamiltonian (3) we now consider the generic behavior in the MI phase. As may be seen in Fig. 7 , the data reveal

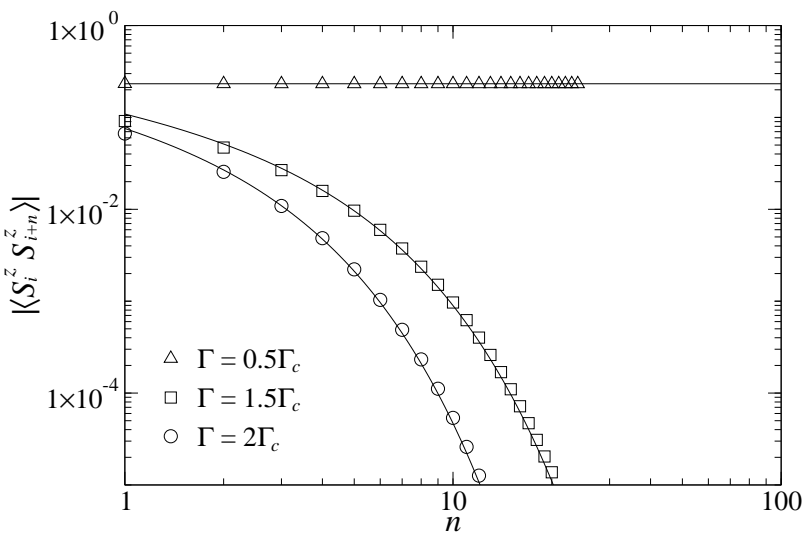

FIG. 10. Off-critical order parameter correlations $\left|\left\langle S_{i}^{z} S_{i+n}^{z}\right\rangle\right|$ obtained by DMRG on the $1 \mathrm{D}$ system (3) with $L=48$ and periodic boundaries. We use the same parameters as in Fig. 5 . The solid lines correspond to the quantum Ising model (4) and are given by Eq. (24) for $\Gamma<\Gamma_{\mathrm{c}}$ and Eq. (25) for $\Gamma>\Gamma_{\mathrm{c}}$. The agreement confirms the presence of long range order for $\Gamma<\Gamma_{\mathrm{c}}$ and exponential decay for $\Gamma>\Gamma_{\mathrm{c}}$.

a finite correlation length for the connected correlation functions on either side of the transition. This is consistent with the presence of an Ising spectral gap as shown in Fig. 3. In order to place this behavior on a quantitative footing we recall the principal results for the transverse field Ising model (4) with $h=0$. In the ordered phase with $\Gamma<\Gamma_{\mathrm{c}}$ the leading contribution to the longitudinal correlations is given by [66]

$$
\left\langle S_{i}^{z} S_{i+n}^{z}\right\rangle \sim(-1)^{n}\left|\left\langle S_{i}^{z}\right\rangle\right|^{2},
$$

where $\left|\left\langle S_{i}^{z}\right\rangle\right|$ is the staggered magnetization corresponding to the onset of long range antiferromagnetic order as given by Eq. (15). In contrast, in the disordered phase with $\Gamma>\Gamma_{\mathrm{c}}$, the correlations decay exponentially with a power law prefactor 66]

$$
\left\langle S_{i}^{z} S_{i+n}^{z}\right\rangle \sim \frac{(-1)^{n}}{4}\left[1-\left(\frac{\Gamma_{\mathrm{c}}}{\Gamma}\right)^{2}\right]^{-1 / 4} \frac{e^{-n / \xi}}{\sqrt{\pi n}},
$$

where $\xi^{-1}=\ln \left(\Gamma / \Gamma_{\mathrm{c}}\right)$. To confirm this behavior we descend deep in to the Mott phase in order to suppress the effects of the small XY terms given by Eq. (14). As shown in Fig. 10 the results are in excellent agreement with the theoretical predictions (24) and (25).

\section{ATOM-MOLECULE CORRELATIONS}

The above considerations are consistent with the notion that away from the Ising transition line the connected correlations decay exponentially in the MI. As advocated in Ref. [32] the absence of low lying gapless excitations precludes the possibility of the novel superMott behavior proposed in Ref. 39, 40]. This gained further support in a recent comment [41] which confirms 
the presence of exponential decay in the atom-molecule correlation functions. We discuss these observations for the bosonic Hamiltonian (3) within the framework of the Ising description.

In order to investigate the possibility of counterflow supercurrents [64, 65] of atoms and molecules, the authors of Ref. [41] consider the connected correlation function

$$
\begin{aligned}
C_{a m}(n)= & \left\langle m^{\dagger}(n) a(n) a(n) a^{\dagger}(0) a^{\dagger}(0) m(0)\right\rangle \\
& -\left\langle m^{\dagger}(n) a(n) a(n)\right\rangle\left\langle a^{\dagger}(0) a^{\dagger}(0) m(0)\right\rangle .
\end{aligned}
$$

Using the spin mapping (5) 32, 33 this may be written in the equivalent form

$$
C_{a m}(n)=2\left[\left\langle S^{+}(n) S^{-}(0)\right\rangle-\left\langle S^{+}(n)\right\rangle\left\langle S^{-}(0)\right\rangle\right] .
$$

Deep within the second Mott lobe one may therefore use the Ising Hamiltonian (4) to gain a handle on the bosonic correlation function (26). To this end we may decompose the first term in Eq. (27) as

$$
\left\langle S_{i}^{+} S_{i+n}^{-}\right\rangle=\left\langle S_{i}^{x} S_{i+n}^{x}\right\rangle+\left\langle S_{i}^{y} S_{i+n}^{y}\right\rangle,
$$

where the mixed component terms cancel. It follows that

$$
C_{a m}(n)=2\left[\left\langle S_{i}^{x} S_{i+n}^{x}\right\rangle-\left\langle S_{i}^{x}\right\rangle\left\langle S_{i+n}^{x}\right\rangle+\left\langle S_{i}^{y} S_{i+n}^{y}\right\rangle\right],
$$

where we use the fact that $\left\langle S_{i}^{y}\right\rangle=0$ for the transverse field Ising model (4) in zero magnetic field. That is to say, $C_{a m}(n)$ is the sum of the connected $x x$ correlation function and the $y y$ correlation function. From our previous discussion in Sec. VII these contributions generically decay exponentially, in agreement with the findings of Ref. 41]. However, along the locus of the Ising quantum phase transition one expects power law contributions. Using Eqs. (18) and (19) one obtains

$$
C_{a m}(n) \sim 2\left[(2 \pi n)^{-2}-(-1)^{n}(\mathcal{B} / 4) n^{-9 / 4}\right] .
$$

In order to confirm this characteristic oscillatory dependence we perform DMRG on the 1D bosonic Hamiltonian (3) with $L=96$ and periodic boundaries. Employing the finite size result (20) obtained by conformal transformation one obtains

$$
\frac{C_{a m}(n)}{2} \sim \frac{1}{(2 \pi)^{2}}\left[\frac{\pi / L}{\sin \left(\frac{\pi n}{L}\right)}\right]^{2}-(-1)^{n} \frac{\mathcal{B}}{4}\left[\frac{\pi / L}{\sin \left(\frac{\pi n}{L}\right)}\right]^{9 / 4} .
$$

As shown in Fig. 11the results are in excellent agreement with the underlying Ising correlation functions.

\section{CANONICAL SOFTCORE BOSONS}

Having provided a discussion of the model (3) in the reduced Hilbert space, with $r_{a}=2$ and $r_{m}=1$, we turn our attention to the more general problem with canonical softcore bosons. In this situation one must allow for

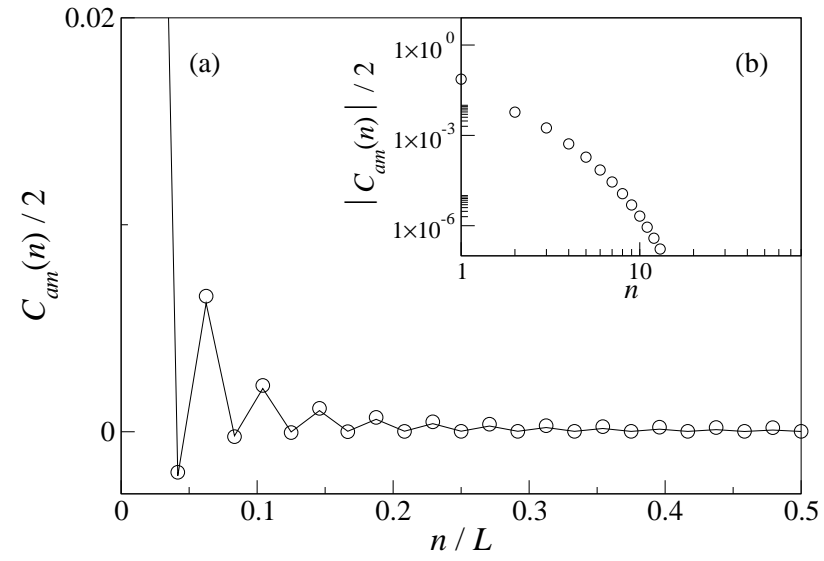

FIG. 11. Correlation function $C_{a m}(n) / 2$ at and away from criticality obtained by DMRG on the $1 \mathrm{D}$ system (3) with $L=48$ and periodic boundaries. We use the same parameters as in Fig. 5 (a) At criticality clear oscillations are present in conformity with the Ising description. The solid line corresponds to the theoretical prediction in Eq. (31). (b) Away from the critical point with $\Gamma=2 \Gamma_{\mathrm{c}}, C_{a m}(n)$ shows a finite correlation length due to the Ising gap.

the presence of additional virtual intermediate states in the magnetic description. For example, in a configuration with two pairs of $a$-atoms on neighboring sites the softcore problem allows virtual hopping to take place, in contrast to the problem with $r_{a}=2$. This modifies the coefficients, $J_{z z}, h$, and $C$, but the Ising description remains valid deep within the second Mott lobe. One again obtains the effective magnetic Hamiltonian given in Eq. (4) but with the modified coefficients,

$$
J_{z z}=\frac{4 t_{a}^{2}}{U_{a m}-U_{a a}}+\frac{t_{m}^{2}}{U_{a m}}-\frac{12 t_{a}^{2}}{U_{a a}}-\frac{4 t_{m}^{2}}{U_{m m}}
$$

and

$$
h=\epsilon_{m}-2 \epsilon_{a}-U_{a a}+\frac{z}{2}\left(\frac{12 t_{a}^{2}}{U_{a a}}-\frac{4 t_{m}^{2}}{U_{m m}}\right),
$$

together with

$$
\begin{aligned}
C & =L\left(\frac{\epsilon_{m}}{2}+\epsilon_{a}+\frac{U_{a a}}{2}\right. \\
& \left.-\frac{z}{8}\left\{\frac{4 t_{a}^{2}}{U_{a m}-U_{a a}}+\frac{t_{m}^{2}}{U_{a m}}+\frac{12 t_{a}^{2}}{U_{a a}}+\frac{4 t_{m}^{2}}{U_{m m}}\right\}\right),
\end{aligned}
$$

as shown in Appendix A. Note in particular that the effective longitudinal magnetic field, $h$, now depends on the hopping parameters and is therefore already present at second order in the strong coupling expansion. In order to see the effect of these additional intermediate states it is instructive to examine the change in the ground state energy of the bosonic Hamiltonian (3) upon increasing the local atomic Hilbert space restriction, $r_{a}$. For simplicity, we consider $U_{m m} \rightarrow \infty$, corresponding to hardcore molecules with $r_{m}=1$. As shown in Fig. 12(a), 


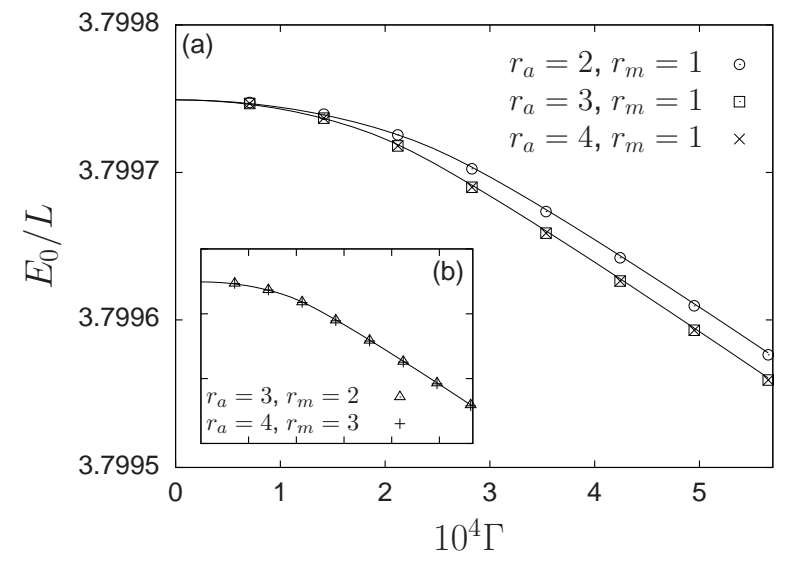

FIG. 12. Ground state energy density of the Hamiltonian (3) obtained by DMRG with $L=20$ (to aid comparison with ED results for the Ising model) and periodic boundaries. (a) We use the parameters in Fig. 5 and increase the local atomic Hilbert space restriction $r_{a}$, with $r_{m}=1$ held fixed. The lines are results for the energy density of the Ising Hamiltonian (4) obtained by $\operatorname{ED}(L=20)$ with $J_{z z}=5.02 \times 10^{-4}$ and $h=0$ for $r_{a}=2$, and $J_{z z}=4.23 \times 10^{-4}$ and $h=7.89 \times 10^{-5}$ for $r_{a}=3,4$. The change from $r_{a}=2$ to $r_{a}=3$ is due to the presence of additional virtual states which modify the Ising model coefficients. The absence of any change beyond $r_{a}=3$ is consistent with second order perturbation theory around the second Mott lobe. (b) Ground state energy density for the same parameters as in panel (a) with the additional interaction $U_{m m}=4$. We increase the local Hilbert space from $r_{a}=3$ and $r_{m}=2$ to $r_{a}=4$ and $r_{m}=3$. The absence of any further change is consistent with the maximum occupancy for virtual states explored in second order perturbation theory around the second Mott lobe. The solid line is the energy density of the Ising Hamiltonian (4) obtained by $\operatorname{ED}(L=20)$ with $J_{z z}=4.16 \times 10^{-4}$ and $h=7.27 \times 10^{-5}$ as given by Eqs. (32) and (33) respectively.

the ground state energy changes on going from $r_{a}=2$ to $r_{a}=3$ due to these additional hopping processes. However, increasing the atomic restriction beyond $r_{a}=3$ has no further effect, since higher occupations are not explored at second order in perturbation theory within the second Mott lobe. The excellent agreement of the bosonic results with $r_{a}=3$ and $r_{a}=4$ therefore supports the applicability of the second order Ising description. The solid lines shown in Fig. 12(a) correspond to the ground state energy density of the Ising model (4) obtained by exact diagonalization with the appropriate coefficients. Our DMRG results are in excellent agreement with these predictions. As shown in Fig. 12(b) this agreement also extends to the generic softcore problem. At second order in perturbation theory around the second Mott lobe the maximum local occupation is $r_{a}=3$ and $r_{m}=2$. The absence of any further change in the ground state energy on increasing the local Hilbert space justifies both the use of this truncation and the Ising description. In Fig. 13 we show the existence of an antiferromagnetic Ising quantum phase transition within the second Mott

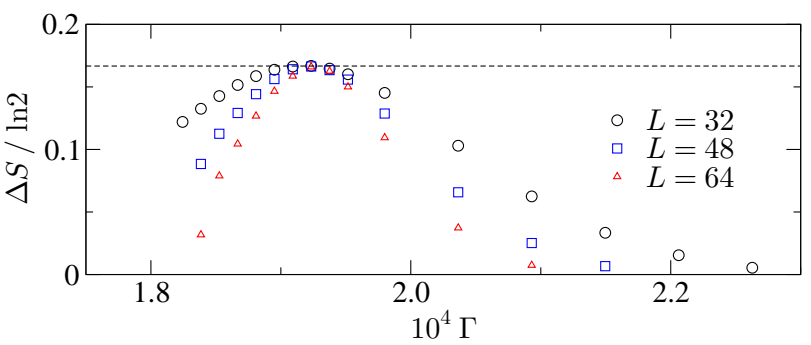

FIG. 13. Entanglement entropy difference $\Delta S(L) \equiv$ $S_{L}(L / 2)-S_{L / 2}(L / 4)$ showing an Ising quantum phase transition within the second Mott lobe for restricted softcore bosons. We truncate the local Hilbert space to $r_{a}=3$ and $r_{m}=2$ which is the maximum occupancy explored at second order in perturbation theory. We use the same parameters as in Fig. 12(b) and set $t=0.005$. The peak height corresponds to a central charge $c \approx 1 / 2$.

lobe for these restricted softcore bosons. The entanglement entropy difference as given by Eq. (12) exhibits a peak on transiting through the magnetic transition. At criticality $\Delta S=\frac{c}{3} \ln 2+\ldots$ and the peak height is in good agreement with an Ising quantum phase transition with central charge $c=1 / 2$.

\section{FERROMAGNETIC ISING TRANSITION}

An interesting aspect of the canonical softcore result (32) is that one may explore both antiferromagnetic and ferromagnetic Ising interactions due to the relative minus signs. This is readily seen by exact diagonalization on small systems using the techniques employed in Refs. [32, 33, 69]. As shown in Fig. 14] an Ising transition indeed persists with ferromagnetic parameters and $h=0$. This is also confirmed by DMRG results for the ground state energy of the bosonic Hamiltonian (3) with $L=64$ and periodic boundary conditions. In the thermodynamic limit, the ground state energy density of the Ising model (4) is given by [66], $E_{0} / L=C / L+e_{\infty}$, where

$$
e_{\infty}=-\frac{1}{4 \pi} \int_{0}^{\pi} d k \sqrt{4 \Gamma^{2}+J_{z z}^{2}+4 \Gamma J_{z z} \cos k}
$$

Defining $E_{0}^{\prime} \equiv E_{0}-C$ the result for $E_{0}^{\prime} / L$ is in good agreement with Eq. (35) as shown in Fig. 15 (a). In addition the finite size corrections to the ground state energy are well described by the conformal result [70, 71]

$$
\frac{E_{0}^{\prime}}{L}=e_{\infty}-\frac{\pi c v}{6 L^{2}}+\ldots
$$

where $v=\left|J_{z z}\right| / 2$ is the effective velocity and $c$ is the central charge. Going beyond our $h=0$ parameter choice, this opens up the exciting possibility of studying the celebrated $\mathrm{E}_{8}$ mass spectrum of the ferromagnetic Ising model in a magnetic field [46]. As exemplified by recent experiments on the quasi-one-dimensional Ising ferromagnet $\mathrm{CoNb}_{2} \mathrm{O}_{6}$ (cobalt niobate) [47, 48], it 

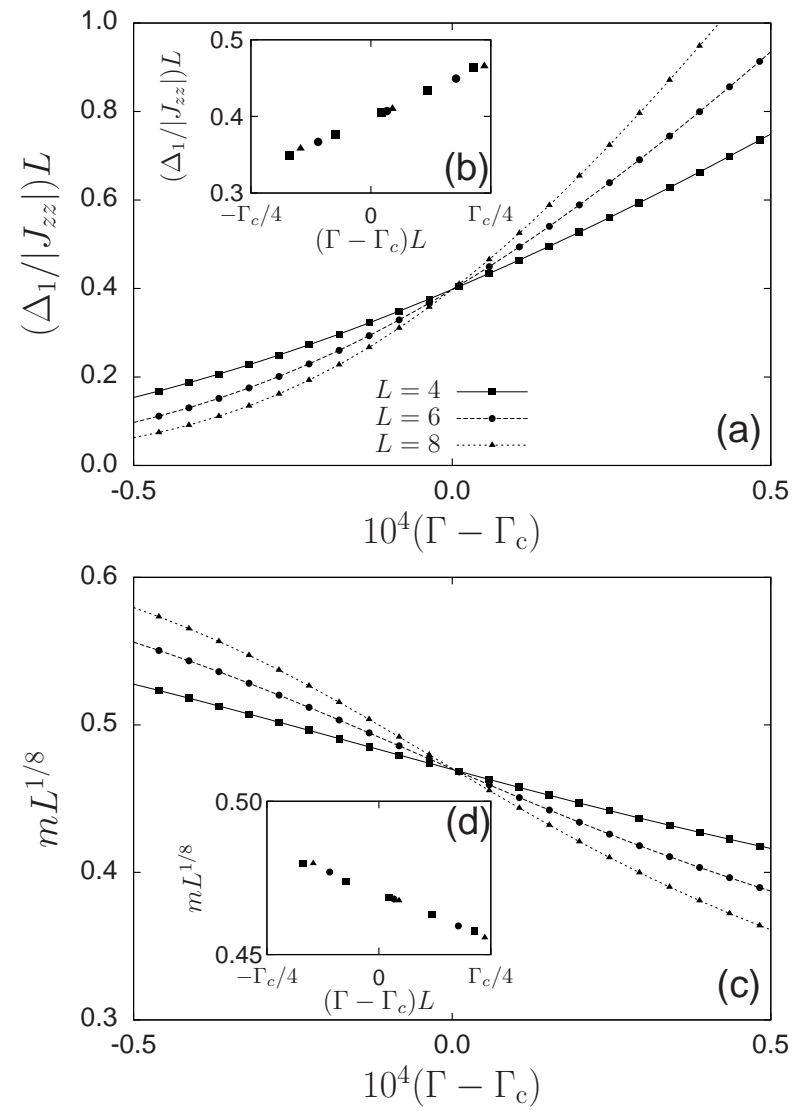

FIG. 14. ED results for the Hamiltonian (3) with hardcore molecules $\left(r_{m}=1\right)$ and up to three atoms $\left(r_{a}=3\right)$ per site. We set $\epsilon_{a}=0, U_{a a}=2, U_{a m}=4, t_{a}=2 t_{m}$ and take $t_{a}=0.01$ corresponding to $\mathrm{FM}$ exchange with $J_{z z} \simeq-3.94 \times 10^{-4}$. For simplicity we set $h=\epsilon_{m}-2+6 t_{a}^{2}=0$ by taking $\epsilon_{m}=1.9994$. (a) The rescaled energy gap $\Delta_{1} \equiv E_{1}-E_{0}$ shows a clear intersection at $\Gamma_{\mathrm{c}} \approx 1.969 \times 10^{-4} \approx 0.4997\left|J_{z z}\right|$ corresponding to a FM transition. (b) Scaling collapse with the Ising critical exponent, $\nu=1$. (c) The rescaled pseudo-magnetization $m=$ $\left\langle\left|\sum_{i} S_{i}^{z}\right|\right\rangle / L$ indicates a transition at the same value of $\Gamma_{\mathrm{c}}$. (d) Scaling collapse with the Ising critical exponent $\beta=1 / 8$.

would be interesting to probe the dynamical correlation functions of the 1D bosonic Hamiltonian (1) in more detail. The non-trivial excitations will manifest themselves at specific frequencies dictated by the emergent $\mathrm{E}_{8}$ mass spectrum. Similar behavior is also expected in the AFM case in the presence of a staggered longitudinal magnetic field.

\section{CONCLUSIONS}

We have investigated the Mott insulating state of bosonic pairing Hamiltonians using analytical and numerical techniques. We have described the behavior of a broad range of physical observables, including local expectation values and correlation functions, within the framework of the paradigmatic quantum Ising model.
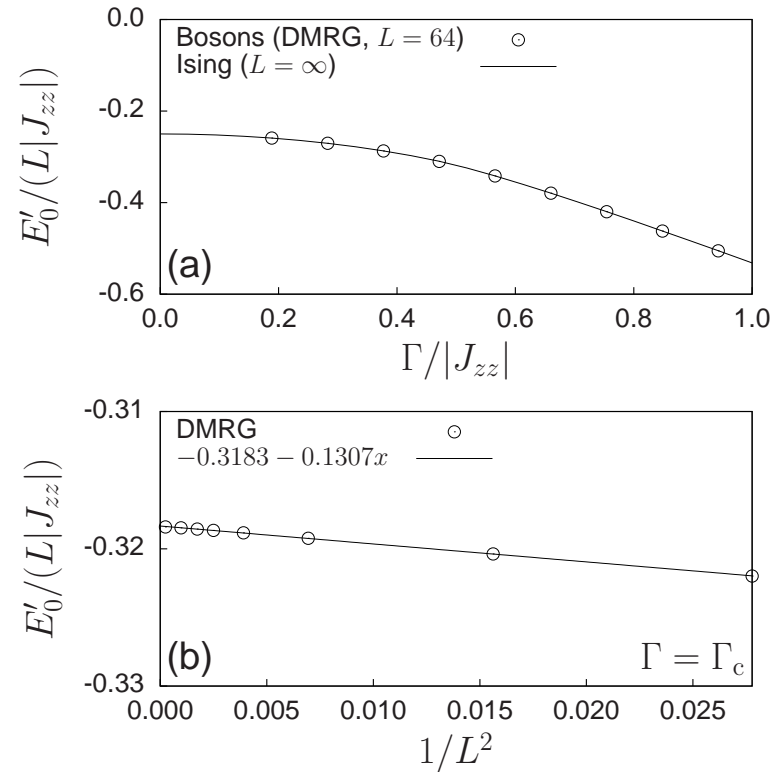

FIG. 15. (a) Rescaled ground state energy $E_{0}^{\prime} \equiv E_{0}-C$ of the bosonic Hamiltonian (3) with the FM parameters used in Fig. 14. The DMRG data are in good agreement with Eq. (35) for the thermodynamic limit of the Ising model. (b) The residual finite size corrections, $E_{0}^{\prime} / L\left|J_{z z}\right|=e_{\infty} /\left|J_{z z}\right|-$ $\pi c / 12 L^{2}+\ldots$, are consistent with the central charge $c \approx$ 0.499 of an Ising transition to a FM state in the absence of a longitudinal magnetic field.

As advocated in Refs. 32, 33, 41] our results are consistent with the absence of super-Mott behavior within the second Mott lobe. Our results differ from the usual two-band Bose-Hubbard model which exhibits counterflow superfluidity [64, 65] since Feshbach resonant pairing interactions favor a distinct Mott phase with either two atoms or one molecule per site. As such, XY terms generically appear at cubic order in the hopping strengths and are intrinsically suppressed. An alternative way to see this is to note that for finite Feshbach coupling, the symmetry of the Hamiltonian is reduced from $\mathrm{U}(1) \times \mathrm{U}(1)$ down to $\mathrm{U}(1) \times \mathbb{Z}_{2}$. As such, one naively expects Ising transitions to occur in a Mott phase with large phase fluctuations and an unbroken U(1) symmetry. Nonetheless, one cannot rule out the possibility of novel transitions in other regions of the phase diagram due to higher order terms in the strong coupling expansion becoming appreciable. It would be interesting to explore this in more detail. In addition, we highlight the possibility of using these systems to explore the $\mathrm{E}_{8}$ mass spectrum of the FM Ising model in a magnetic field. There are many directions for further research including the influence of higher bands and the possibility of realizing Ising transitions in Bose-Fermi mixtures; see Appendix B 


\section{ACKNOWLEDGMENTS}

We are grateful to F. Assaad, J. Oitmaa, F. Pollmann, N. Shannon and A. Tsvelik for helpful discussions. MJB, AOS and BDS acknowledge EPSRC grant no. EP/E018130/1. FHLE by EP/D050952/1. SE and $\mathrm{HF}$ acknowledge funding by the DFG through grant SFB 652. MH by DFG FG1162.

\section{Appendix A: Effective Magnetic Hamiltonian}

To obtain the effective spin description within the second Mott lobe we perform degenerate perturbation theory. We partition the Hamiltonian as $H=H_{0}+V$ where

$$
H_{0}=\sum_{i}\left[H_{i}^{\prime}\left(1-\mathcal{P}_{0 i}\right)+\left(\epsilon_{m}-h_{0} / 2\right) \mathcal{P}_{0 i}\right],
$$

and

$$
V=-\sum_{\langle i j\rangle \alpha} t_{\alpha}\left(b_{i \alpha}^{\dagger} b_{j \alpha}+\text { H.c. }\right)+H_{\mathrm{F}}+h_{0} \sum_{i} S_{i}^{z} \mathcal{P}_{0 i} .
$$

Here $h_{0}=\epsilon_{m}-2 \epsilon_{a}-U_{a a}, \mathcal{P}_{0 i}=|\Uparrow\rangle_{i}\left\langle\left.\Uparrow\right|_{i}+\mid \Downarrow\right\rangle_{i}\left\langle\left.\Downarrow\right|_{i}\right.$ and

$$
H_{i}^{\prime}=\sum_{\alpha} \epsilon_{\alpha} n_{i \alpha}+\sum_{\alpha \alpha^{\prime}} \frac{U_{\alpha \alpha^{\prime}}}{2}: n_{i \alpha} n_{i \alpha^{\prime}}:
$$

This approach is appropriate deep in the Mott lobe and in the vicinity of the transition where $g, h_{0}$ and $t^{2} / U$ are small and $V$ may be treated perturbatively. Up to second order in $V$ the effective Hamiltonian is

$H_{\text {eff }}=\mathcal{P}_{0}\left(H_{0}+V+V\left(1-\mathcal{P}_{0}\right) \frac{1}{E_{0}-H_{0}}\left(1-\mathcal{P}_{0}\right) V\right) \mathcal{P}_{0}$

where $E_{0}=L\left(\epsilon_{m}-h_{0} / 2\right)$ and $\mathcal{P}_{0}=\prod_{i} \mathcal{P}_{0 i}$. Since the hopping terms are the only source of coupling between the degenerate subspace spanned by $\mathcal{P}_{0}$ and the remaining Hilbert space, the second order term reduces to

$$
H_{\mathrm{eff}}^{(2)}=\sum_{\langle i j\rangle \alpha} t_{\alpha}^{2} \mathcal{P}_{0} b_{i \alpha}^{\dagger} b_{j \alpha} \frac{1}{E_{0}-H_{0}} b_{j \alpha}^{\dagger} b_{i \alpha} \mathcal{P}_{0}+\text { H.c.. }
$$

This acts on two sites so within the degenerate subspace $\mathcal{P}_{0}$, we must consider its action on four basis states. The action of $H_{\mathrm{eff}}^{(2)}$ on sites with neighboring molecules yields

$$
H_{\mathrm{eff}}^{(2)}|\Uparrow\rangle_{i}|\Uparrow\rangle_{j}=-\frac{4 t_{m}^{2}}{U_{m m}+h_{0}}|\Uparrow\rangle_{i}|\Uparrow\rangle_{j}
$$

and for an arrangement of neighboring atoms gives

$$
H_{\mathrm{eff}}^{(2)}|\Downarrow\rangle_{i}|\Downarrow\rangle_{j}=-\frac{12 t_{a}^{2}}{U_{a a}-h_{0}}|\Downarrow\rangle_{i}|\Downarrow\rangle_{j}
$$

When the neighboring species are different

$$
H_{\mathrm{eff}}^{(2)}|\Uparrow\rangle_{i}|\Downarrow\rangle_{j}=-\left(\frac{2 t_{a}^{2}}{U_{a m}-U_{a a}}+\frac{t_{m}^{2}}{2 U_{a m}}\right)|\Uparrow\rangle_{i}|\Downarrow\rangle_{j} .
$$

In order to obtain the second order contribution to the effective Hamiltonian we collate these terms. In addition we exploit the spin projection identities $|\Uparrow\rangle_{i}\left\langle\left.\Uparrow\right|_{i} \mathcal{P}_{0}=\right.$ $\left(1 / 2+S_{i}^{z}\right) \mathcal{P}_{0}$ and $|\Downarrow\rangle_{i}\left\langle\left.\Downarrow\right|_{i} \mathcal{P}_{0}=\left(1 / 2-S_{i}^{z}\right) \mathcal{P}_{0}\right.$ and expand the resulting expression to leading (zeroth) order in $h_{0}$ :

$$
H_{\mathrm{eff}}^{(2)} \simeq \mathcal{P}_{0}\left(J_{z z} \sum_{\langle i j\rangle} S_{i}^{z} S_{j}^{z}+h_{2} \sum_{i} S_{i}^{z}+C_{2}\right) \mathcal{P}_{0}
$$

where $J_{z z}$ is given by Eq. (32) and

$$
h_{2}=\frac{z}{2}\left(\frac{12 t_{a}^{2}}{U_{a a}}-\frac{4 t_{m}^{2}}{U_{m m}}\right) .
$$

The constant offset is given by

$$
C_{2}=-\frac{z L}{8}\left(\frac{4 t_{a}^{2}}{U_{a m}-U_{a a}}+\frac{t_{m}^{2}}{U_{a m}}+\frac{12 t_{a}^{2}}{U_{a a}}+\frac{4 t_{m}^{2}}{U_{m m}}\right) .
$$

Inclusion of the zeroth and first order contributions in $V$ yields the effective Ising Hamiltonian (4) with $h=h_{0}+h_{2}$ and $C=E_{0}+C_{2}$ as given by Eqs. (33) and (34). In order to obtain the effective Hamiltonian with $r_{a}=2$ one must exclude the contribution from Eq. (A7) as the intermediate states involved in the perturbation do not satisfy the imposed constraint. Similarly if $r_{m}=1$ the contribution from Eq. (A6) must be excluded.

\section{Appendix B: Bose-Fermi Hamiltonian}

Throughout this manuscript we have focused exclusively on the bosonic homonuclear Hamiltonian (1) and the associated Ising description. However, it is evident from the general setup shown in Fig. 1 that similar results may also emerge with more than one atomic species. For example, this is confirmed in Refs. [32, 33] for the heteronuclear bosonic case. In this appendix we note that a similar Ising description may also apply with twocomponent fermionic atoms and bosonic molecules. We consider the Bose-Fermi Hamiltonian

$$
\begin{aligned}
H & =\sum_{i \alpha} \epsilon_{\alpha} n_{i \alpha}-\sum_{\langle i j\rangle} \sum_{\alpha} t_{\alpha}\left(d_{i \alpha}^{\dagger} d_{j \alpha}+\text { H.c. }\right)+H_{\mathrm{F}} \\
& +\sum_{i, \alpha \neq \alpha^{\prime}} \frac{U_{\alpha \alpha^{\prime}}}{2} n_{i \alpha} n_{i \alpha^{\prime}}+\sum_{i} \frac{U_{m m}}{2} n_{i m}\left(n_{i m}-1\right),
\end{aligned}
$$

where $\alpha=\downarrow, \uparrow$ are fermionic atoms, $\alpha=m$ is a bosonic molecule, $n_{i \alpha}=d_{i \alpha}^{\dagger} d_{i \alpha}$ and $H_{\mathrm{F}}=g \sum_{i}\left(d_{i m}^{\dagger} d_{i \downarrow} d_{i \uparrow}+\right.$ H.c. $)$. Assuming the existence of a second Mott lobe as depicted in Fig. 1] with either two fermionic atoms or a bosonic molecule per site, one again obtains an Ising Hamiltonian (44) acting on the states $|\Downarrow\rangle=d_{\uparrow}^{\dagger} d_{\downarrow}^{\dagger}|0\rangle$ and $|\Uparrow\rangle=d_{m}^{\dagger}|0\rangle$. The parameters in Eq. (4) are now given by $\Gamma=2 g$ and

$$
\frac{J_{z z}}{2}=\frac{t_{m}^{2}}{U_{\uparrow m}+U_{\downarrow m}}+\frac{t_{\downarrow}^{2}}{U_{\downarrow m}-U_{\uparrow \downarrow}}+\frac{t_{\uparrow}^{2}}{U_{\uparrow m}-U_{\uparrow \downarrow}}-\frac{2 t_{m}^{2}}{U_{m m}} .
$$


The effective magnetic field is given by

$$
h=\epsilon_{m}-\left(\epsilon_{\downarrow}+\epsilon_{\uparrow}+U_{\uparrow \downarrow}\right)-\frac{2 z t_{m}^{2}}{U_{m m}},
$$

and the constant offset is

$$
C=L\left(\epsilon_{m}-\frac{h}{2}+\frac{z J}{8}-\frac{z t_{m}^{2}}{U_{m m}}\right) .
$$

In the limit $U_{m m} \rightarrow \infty$ the results (B2), (B3) and (B4) coincide with the results of Sec. III where we identify $U_{\uparrow \downarrow}=U_{a a}$ and $U_{\downarrow m}=U_{\uparrow m}=U_{a m}$. In view of our findings in the bosonic problems it would be interesting to examine the Bose-Fermi mixture (B1) in more detail.
[1] M. H. Anderson, J. R. Ensher, M. R. Matthews, C. E. Wieman, and E. A. Cornell, Science, 269, 198 (1995).

[2] K. B. Davis, M.-O. Mewes, M. R. Andrews, N. J. van Druten, D. S. Durfee, D. M. Kurn, and W. Ketterle, Phys. Rev. Lett., 75, 3969 (1995).

[3] D. Jaksch, C. Bruder, J. I. Cirac, C. W. Gardiner, and P. Zoller, Phys. Rev. Lett., 81, 3108 (1998).

[4] M. Greiner, O. Mandel, T. Esslinger, T. W. Hänsch, and I. Bloch, Nature, 415, 39 (2002).

[5] M. P. A. Fisher, P. B. Weichman, G. Grinstein, and D. S. Fisher, Phys. Rev. B, 40, 546 (1989).

[6] J. Stenger, S. Inouye, D. M. Stamper-Kurn, H.-J. Miesner, A. P. Chikkatur, and W. Ketterle, Nature, 396, 345 (1998).

[7] H.-J. Miesner, D. M. Stamper-Kurn, J. Stenger, S. Inouye, A. P. Chikkatur, and W. Ketterle, Phys. Rev. Lett., 82, 2228 (1999).

[8] D. M. Stamper-Kurn, H.-J. Miesner, A. P. Chikkatur, S. Inouye, J. Stenger, and W. Ketterle, Phys. Rev. Lett., 83, 661 (1999).

[9] A. E. Leanhardt, Y. Shin, D. Kielpinski, D. E. Pritchard, and W. Ketterle, Phys. Rev. Lett., 90, 140403 (2003).

[10] T. Ohmi and K. Machida, J. Phys. Soc. Jpn., 67, 1822 (1998).

[11] T.-L. Ho, Phys. Rev. Lett., 81, 742 (1998).

[12] E. Demler and F. Zhou, Phys. Rev. Lett., 88, 163001 (2002).

[13] A. Imambekov, M. Lukin, and E. Demler, Phys. Rev. A, 68, 063602 (2003).

[14] A. Lamacraft, Phys. Rev. Lett., 98, 160404 (2007).

[15] S. Mukerjee, C. Xu, and J. E. Moore, Phys. Rev. B, 76, 104519 (2007).

[16] F. H. L. Essler, G. V. Shlyapnikov, and A. M. Tsvelik, J. Stat. Mech., P02027 (2009).

[17] M. Ueda and Y. Kawaguchi, arXiv:1001.2072.

[18] G. Thalhammer, G. Barontini, L. D. Sarlo, J. Catani, F. Minardi, and M. Inguscio, Phys. Rev. Lett., 100, 210402 (2008).

[19] M. A. Cazallila and A. F. Ho, Phys. Rev. Lett, 91, 150403 (2003).

[20] A. Kuklov, N. Prokof'ev, and B. Svistunov, Phys. Rev. Lett., 92, 050402 (2004).

[21] A. Kleine, C. Kollath, I. P. McCulloch, T. Giamarchi, and U. Schollwöck, Phys. Rev. A, 77, 013607 (2008); New J. Phys., 10, 045025 (2008).

[22] S. G. Söyler, B. Capogrosso-Sansone, N. V. Prokof'ev, and B. V. Svistunov, New J. Phys., 11, 073036 (2009).

[23] M. Guglielmino, V. Penna, and B. Capogrosso-Sansone, Phys. Rev. A, 82, 021601 (2010).
[24] L. M. Duan, E. Demler, and M. D. Lukin, Phys. Rev. Lett., 91, 090402 (2003).

[25] E. Altman, W. Hofstetter, E. Demler, and M. D. Lukin, New J. Phys., 5, 113 (2003).

[26] A. Hubener, M. Snoek, and W. Hofstetter, Phys. Rev. B, 80, 245109 (2009).

[27] S. Powell, Phys. Rev. A, 79, 053614 (2009).

[28] B. Capogrosso-Sansone, S.. G. Söyler, N. V. Prokof'ev, and B. V. Svistunov, Phys. Rev. A, 81, 053622 (2010).

[29] A. V. Gorshkov, M. Hermele, V. Gurarie, C. Xu, P. S. Julienne, J. Ye, P. Zoller, E. Demler, M. D. Lukin, and A. M. Rey, Nat. Phys., 6, 289 (2010).

[30] M. Hermele, V. Gurarie, and A. M. Rey, Phys. Rev. Lett., 103, 135301 (2009).

[31] M. A. Cazalilla, A. F. Ho, and M. Ueda, New J. Phys., 11, 103033 (2009).

[32] M. J. Bhaseen, A. O. Silver, M. Hohenadler, and B. D. Simons, Phys. Rev. Lett., 103, 265302 (2009).

[33] M. Hohenadler, A. O. Silver, M. J. Bhaseen, and B. D. Simons, Phys. Rev. A, 82, 013639 (2010).

[34] L. Radzihovsky, J. Park, and P. B. Weichman, Phys. Rev. Lett., 92, 160402 (2004).

[35] M. W. J. Romans, R. A. Duine, S. Sachdev, and H. T. C. Stoof, Phys. Rev. Lett., 93, 020405 (2004).

[36] L. Radzihovsky, P. B. Weichman, and J. I. Park, Ann. Phys., 323, 2376 (2008).

[37] D. B. M. Dickerscheid, U. Al Khawaja, D. van Oosten, and H. T. C. Stoof, Phys. Rev. A, 71, 043604 (2005).

[38] K. Sengupta and N. Dupuis, Europhys. Lett., 70, 586 (2005).

[39] V. G. Rousseau and P. J. H. Denteneer, Phys. Rev. Lett, 102, 015301 (2009).

[40] V. G. Rousseau and P. J. H. Denteneer, Phys. Rev. A, 77, 013609 (2008).

[41] M. Eckholt and T. Roscilde, Phys. Rev. Lett., 105, 199603 (2010).

[42] S. Ejima, M. J. Bhaseen, M. Hohenadler, F. H. L. Essler, H. Fehske, and B. D. Simons, Phys. Rev. Lett., 106, 015303 (2011).

[43] S. R. White, Phys. Rev. Lett., 69, 2863 (1992); Phys. Rev. B, 48, 10345 (1993).

[44] B. M. McCoy and T. T. Wu, Phys. Rev. D, 18, 1259 (1978).

[45] M. J. Bhaseen and A. M. Tsvelik, in From Fields To Strings: Circumnavigating Theoretical Physics, Ian Kogan Memorial, Vol. I, edited by M. Shifman, A. Vainshtein, and J. Wheater (World Scientific, 2005) pp. 661683. 
[46] A. B. Zamolodchikov, Int. J. Mod. Phys. A, 4, 4235 (1989).

[47] R. Coldea, D. A. Tennant, E. M. Wheeler, E. Wawrzynska, D. Prabhakaran, M. Telling, K. Habicht, P. Smeibidl, and K. Kiefer, Science, 327, 177 (2010).

[48] J. A. Kjäll, F. Pollmann, and J. E. Moore, Phys. Rev. B, 83, 020407 (2011).

[49] L. Radzihovsky and S. Choi, Phys. Rev. Lett., 103, 095302 (2009).

[50] R. B. Diener and T.-L. Ho, Phys. Rev. A, 73, 017601 (2006).

[51] D. B. M. Dickerscheid, D. van Oosten, and H. T. C. Stoof, Phys. Rev. A, 73, 017602 (2006).

[52] H. P. Büchler, Phys. Rev. Lett., 104, 090402 (2010).

[53] A. J. Daley, J. M. Taylor, S. Diehl, M. Baranov, and P. Zoller, Phys. Rev. Lett., 102, 040402 (2009); 102, 179902 (2009); S. Diehl, M. Baranov, A. J. Daley, and P. Zoller, 104, 165301 (2010).

[54] S. Diehl, M. Baranov, A. J. Daley, and P. Zoller, Phys. Rev. B, 82, 064509 (2010); 82, 064510 (2010).

[55] C. Wu, Phys. Rev. Lett., 95, 266404 (2005); P. Lecheminant, E. Boulat, and P. Azaria, 95, 240402 (2005).

[56] S. Capponi, G. Roux, P. Azaria, E. Boulat, and P. Lecheminant, Phys. Rev. B, 75, 100503(R) (2007); G. Roux, S. Capponi, P. Lecheminant, and P. Azaria, Eur. Phys. J. B, 68, 293 (2009).

[57] L. Bonnes and S. Wessel, Phys. Rev. Lett., 106, 185302 (2011).
[58] S. Sachdev, Quantum Phase Transitions (Cambridge University Press, 1999).

[59] C. Holzey, F. Larsen, and F. Wilczek, Nucl. Phys. B, 424, 443 (1994).

[60] V. E. Korepin, Phys. Rev. Lett., 92, 096402 (2004).

[61] P. Calabrese and J. Cardy, J. Stat. Mech., P06002 (2004).

[62] O. Legeza, J. Sólyom, L. Tincani, and R. M. Noack, Phys. Rev. Lett., 99, 087203 (2007).

[63] A. M. Läuchli and C. Kollath, J. Stat. Mech., P05018 (2008).

[64] A. B. Kuklov and B. V. Svistunov, Phys. Rev. Lett., 90, 100401 (2003).

[65] A. Hu, L. Mathey, I. Danshita, E. Tiesinga, C. J. Williams, and C. W. Clark., Phys. Rev. A, 80, 023619 (2009).

[66] P. Pfeuty, Ann. Phys., 57, 79 (1970).

[67] Y. Hieida, K. Okunishi, and Y. Akutsu, Phys. Rev. B, 64, 224422 (2001).

[68] J. Cardy, Scaling and Renormalization in Statistical Physics (Cambridge University Press, 1996).

[69] J. Um, S.-I. Lee, and B. J. Kim, J. Korean Phys. Soc., 50, 285 (2007).

[70] H. W. J. Blöte, J. L. Cardy, and M. P. Nightingale, Phys. Rev. Lett., 56, 742 (1986).

[71] I. Affleck, Phys. Rev. Lett., 56, 746 (1986). 